Malaya Journal of Matematik, Vol. S, No. 1, 457-461, 2019

https://doi.org/10.26637/MJM0S01/0081

\title{
Transitively tracked graphs
}

\author{
Jalsiya $\mathrm{M} \mathrm{P}^{1 *}$ and Raji Pilakkat ${ }^{2}$
}

\begin{abstract} graphs.

\section{Keywords}

Cycle tracking set, cycle tracking number $\tau(G)$, transitively tracked graph.

\section{AMS Subject Classification}

05C.

${ }^{1}$ Department of Mathematics, T M Govt College, Tirur, kerala-676502, India.

${ }^{2}$ Department of Mathematics, University of Calicut, Malappuram, Kerala - 673635, India.

*Corresponding author: ${ }^{\text {* }}$ jalsiyajamal@gmail.com; ${ }^{2}$ rajiunical@rediffmail.com

Article History: Received 24 January 2019; Accepted 24 May 2019
\end{abstract}

Let $G(V, E)$ be a graph with vertex set $\mathrm{V}$ and edge set $\mathrm{E}$. For $v \in V(G)$, the cycle trace of $v$ is defined as the set of all vertices $u \in V$ such that $u$ and $v$ belong to same cycle and is denoted by $T_{G}(v)$. For $u, v \in V, v$ is said to be cycle traced by $u$ if $v \in T_{G}(u)$. A set $S$ of vertices in a graph $G(V, E)$ is called a cycle tracking set if for every vertex $v \in V \backslash S$, there exists a vertex $u \in S$ such that $v \in T_{G}(u)$. For every pair of vertices $u, v \in V$, we say that $u$ is related to $v(u \sim v)$ if $u \in T_{G}(v)$. A graph $\mathrm{G}(\mathrm{V}, \mathrm{E})$ is said to be transitively tracked graph if the relation $\sim$ is transitive.

This paper characterizes cycle tracking transitively tracked graphs and determine cycle tracking sets for such

\section{Contents}

1 Transitive tracking Graph .457

References ...........................460

The concept of cycle tracking set was introduced by Jalsiya and Raji Pilakkat [3]. For notation and graph theory terminology we in general follow $[1,8]$. Let $G(V, E)$ be a graph. For $v \in V(G)$, the cycle trace (simply trace) of $v$ is defined as the set of all vertices $u \in V$ such that $u$ and $v$ belong to same cycle of $\mathrm{G}$ and is denoted by $T_{G}(v)$. For $u, v \in V, v$ is said to be cycle traced (or simplytraced) by $u$ if $v \in T_{G}(u)$. A set $\mathrm{S}$ of vertices in a graph $G(V, E)$ is called a cycle tracking set if for every vertex $v \in V \backslash S$, there exists a vertex $u \in S$ such that $v \in T_{G}(u)$. A cycle tracking set is a minimal cycle tracking set if no proper subset $S^{\prime} \subset S$ is a cycle tracking set. The cycle tracking number $\tau(G)$ of a graph $\mathrm{G}$ is the minimum cardinality of a minimal cycle tracking set of $\mathrm{G}$. The upper cycle tracking number $T(G)$ of a graph $\mathrm{G}$ is the maximum cardinality of minimal cycle tracking set of G. A cycle tracking set with minimum cardinality is called a $\tau-$ set of $G$.

In [4] Jalsiya and Raji pilakkat introduced the concept of independent cycle tracking set. Two vertices $\mathrm{u}, \mathrm{v}$ of a graph $\mathrm{G}$ are said to be trace independent if $u \notin T_{G}(v)$. A set $\mathrm{S}$ of vertices in a graph $G(V, E)$ is called a trace independent set if any two vertices of $S$ are trace independent in G. If a cycle tracking set $S$ is trace independent then $S$ is called independent cycle tracking set. The minimum cardinality of an independent cycle tracking set of $\mathrm{G}$ is the independent cycle tracking number and is denoted by $\tau_{i}(G)$. The maximum cardinality of independent cycle tracking set of $\mathrm{G}$ is called the upper independent cycle tracking number and is denoted by $T_{i}(G)$.

A graph $\mathrm{G}(\mathrm{V}, \mathrm{E})$ is said to be track connected if for every pair of vertices $u, v \in V(G)$ there exists two internally disjoint paths connecting $\mathrm{u}$ and $\mathrm{v}$. An induced subgraph of a graph $\mathrm{G}$ is said to be maximal track connected if it is not a proper subgraph of any track connected subgraph of G. A vertex is said to be trace free vertex if it belongs to no cycle. In this paper we characterizes transitively tracked graphs and determine cycle tracking set for such graphs. Through out this paper we consider simple finite and undirected graphs.

\section{Transitive tracking Graph}

In [3] Jalsiya and Raji pilakkat obtained the following result for track connected graph.

Theorem 1.1. [3] If graph $G$ is track connected then $\tau(G)=$ 1.

A generalization of the above theorem is possible for a particular type of graphs. Such graphs are introduced by 
introducing a relation $\sim$ on the vertex set $\mathrm{V}$ of a graph $\mathrm{G}$ as follows: For every pair of vertices $u, v \in V$, we say that $\mathrm{u}$ is related to $\mathrm{v}(u \sim v)$ if $u \in T_{G}(v)$. Then $\sim$ is a reflexive and symmetric relation

Definition 1.2. A graph $G(V, E)$ is said to be transitively tracked graph if the relation $\sim$ is transitive. ie; for every triple of vertices $u, v, w \in V, w \in T_{G}(u)$ and $u \in T_{G}(v)$ implies $w \in T_{G}(v)$.

Every track connected graph is transitively tracked.

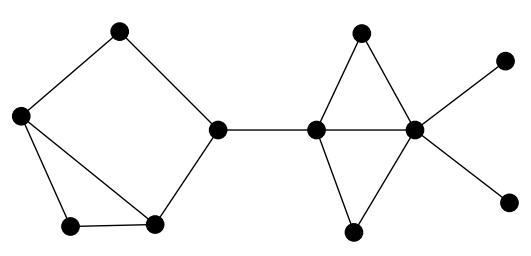

G

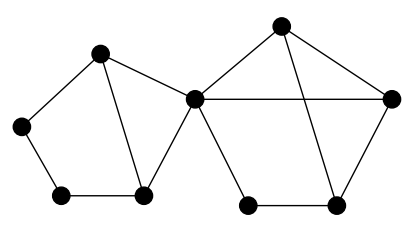

$\mathrm{H}$

Figure 1. $\mathrm{G}$ is a transitively tracked graph but $\mathrm{H}$ is not.

Theorem 1.3. A graph $G(V, E)$ is a transitively tracked graph if only if $V(G)$ can be partitioned into $V_{1}, V_{2}, \ldots, V_{k}$ such that the graph $\left\langle V_{i}\right\rangle$ induced by each $V_{i}$ is a maximal track connected subgraph of $G$.

Proof. Let $\mathrm{G}(\mathrm{V}, \mathrm{E})$ be a transitively tracked graph. Then the relation $\sim$ defined on the vertex set $\mathrm{V}$ of $\mathrm{G}$ by $u \sim v$ if $v \in T_{G}(u)$ is an equivalence relation. Hence there exists a partition of $\mathrm{V}(\mathrm{G})$ into equivalence classes. We denote the equivalence class containing $v$ by $[v]$

Let $v \in V$. Suppose $u \in[v]$. Then $u \sim v$ and hence $v \in T_{G}(u)$ ie; $u$ and $v$ belong to same cycle. That is there exist two internally disjoint paths from $\mathrm{u}$ to $\mathrm{v}$. Therefore the graph induced by $[v]$ is track connected.

If the graph induced by $[v]$ is not a maximal track connected subgraph. Then there exists a vertex $w \notin[v]$ such that $w$ and $v$ belong to same cycle. Therefore $w \in T_{G}(v)$. Hence $w \sim v$, a contradiction. Therefore the graph induced by $[v]$ is a maximal track connected subgraph.

Conversely suppose that $V(G)$ can be partitioned into $V_{1}, V_{2}, \ldots, V$ such that the graph induced by each $V_{i}$ is a maximal track connected subgraph of G. Let $u, v, w \in V(G)$ such that $u \in T_{G}(v)$ and $w \in T_{G}(u)$. Then $u, v \in V_{i}$ for some $\mathrm{i}$ and $u, w \in V_{j}$ for some j. Since $V_{i}$ and $V_{j}$ are disjoint if $i \neq j$, we conclude that $i=j$. That is $\mathrm{u}, \mathrm{v}, \mathrm{w}$ belong to the same $V_{i}$ and hence $w \in T_{G}(v)$. Therefore $\mathrm{G}$ is a transitively tracked graph.

Transitively tracked graphs can be characterized as follows.

Theorem 1.4. Let $G$ be a graph. A necessary and sufficient condition for $G$ to be a transitively tracked graph is that for any two vertices $u$ and $v$ of $G, v \in T_{G}(u)$ if and only if $T_{G}(v)=T_{G}(u)$.

Proof. Suppose $\mathrm{G}$ is a transitively tracked graph and $u, v \in$ $V(G)$ such that $v \in T_{G}(u)$. Let $w \in T_{G}(v)$. Then $w \in T_{G}(u)$, since $v \in T_{G}(u)$ and $\mathrm{G}$ is a transitively tracked graph. Therefore $T_{G}(v) \subseteq T_{G}(u)$.

On other hand, let $x \in T_{G}(u)$. Since $v \in T_{G}(u)$, we have $u \in T_{G}(v)$. Therefore $x \in T_{G}(v)$. Therefore $T_{G}(u) \subseteq T_{G}(v)$. Thus $T_{G}(v)=T_{G}(u)$.

Conversely suppose that $v \in T_{G}(u)$ if and only if $T_{G}(v)=$ $T_{G}(u)$ for all $u, v \in G$. Let $u, v, w \in G(V)$ such that $u \in T_{G}(v)$ and $w \in T_{G}(u)$. Then $T_{G}(v)=T_{G}(u)=T_{G}(w)$. Hence $w \in$ $T_{G}(v)$. Therefore $\mathrm{G}$ is a transitively tracked graph

Corollary 1.5. Let $S$ be a $\tau$-set of a transitively tracked graph. Then $\sum_{u \in S}\left|T_{G}(u)\right|=|V|$.

Lemma 1.6. If the graph induced by $T_{G}(v)$ is track connected then it is maximal track connected.

Proof. Suppose the graph induced by $T_{G}(v)$ is track connected. If possible assume that $T_{G}(v)$ is not maximal track connected. Then there exists a track connected subgraph $H$ of $G$ such that $T_{G}(v) \subset V(H)$. Let $u \in V(H) \backslash T_{G}(v)$. Since $\mathrm{H}$ is track connected there exist two internally disjoint paths form $u$ to $v$. That is $u \in T_{G}(v)$, a contradiction.

Theorem 1.7. Let $G$ be a transitively tracked graph. Then for every vertex $v$ in $G$, the graph induced by $T_{G}(v)$ is a maximal track connected subgraph of $G$.

Proof. Let $\mathrm{G}$ be a transitively tracked graph. Let $v \in V(G)$. If possible assume that $T_{G}(v)$ not track connected. Then there exist two vertices $u, w \in T_{G}(v)$ such that $u \notin T_{G}(w)$. Since G is transitively tracked, $u \in T_{G}(v)$ and $v \in T_{G}(w)$ implies that $u \in T_{G}(w)$, a contradiction. Hence $T_{G}(v)$ is track connected. By Lemma 1.6 the graph induced by $T_{G}(v)$ is a maximal track connected subgraph of $\mathrm{G}$.

Corollary 1.8. Let $G$ be a transitively tracked graph and let $H$ be a maximal track connected subgraph of $G$. Then for every $v \in V(H), T_{G}(v)=V(H)$.

Proof. Let $\mathrm{G}$ be a transitively tracked graph. Let $H$ be a maximal track connected subgraph of G. Let $v \in V(H)$. Since $H$ is track connected, $H$ is a subgraph of the graph induced by $T_{G}(v)$. Since $\mathrm{G}$ is transitively tracked the graph induced by $T_{G}(v)$ is a track connected subgraph of G. By maximality of $H, V(H)=T_{G}(v)$. Since $v$ is arbitrary $T_{G}(v)=V(H)$ for every vertex in $V(H)$.

Corollary 1.9. Let $G$ be a transitively tracked graph. Then for every vertex $v$ in $G$ there exist a cycle tracking set containing $v$. 
Proof. Let $\mathrm{G}$ be a transitively tracked graph and let $\mathrm{S}$ be any cycle tracking set of G. Let $v$ be a vertex of G. Then there exists a vertex $u$ in $S$ such that $v \in T_{G}(u)$. Then by theorem $1.4 T_{G}(v)=T_{G}(u)$. Let $w \in V$.

case (i) $w \in T_{G}(u)$

. $\quad$ Then $w \in T_{G}(v)$.

case(ii) $w \notin T_{G}(u)$

$T_{G}(x)$.

So $S \backslash\{u\} \cup\{v\}$ forms a cycle tracking set for $\mathrm{G}$ containing $v$.

Theorem 1.10. For a transitively tracked graph $G, \tau(G)=$ number of maximal track connected subgraph of $G$.

Proof. Let $\mathrm{G}$ be a transitively tracked graph. Then $V(G)$ can be partitioned into $V_{1}, V_{2}, \ldots, V_{k}$ such that the graph $\left\langle V_{i}\right\rangle$ induced by each $V_{i}$ is a maximal track connected subgraph of G. Let $\mathrm{S}$ be any $\tau$-set of G. Let $v_{i} \in V_{i}$. Then there exist a $u_{i} \in S$ such that $u_{i} \in T_{G}\left(v_{i}\right)$. Since $\mathrm{G}$ is transitively tracked $T_{G}\left(u_{i}\right)=T_{G}\left(v_{i}\right)=V_{i}$. So for every $i, 1 \leq i \leq k$ there exist $u_{i} \in S$ such that $T_{G}\left(u_{i}\right)=V_{i}$. So $|S| \geq k$. But since $S^{*}=\left\{v_{i}\right.$ : $\left.v_{i} \in V_{i} 1 \leq i \leq k\right\}$ is a cycle tracking set of $\mathrm{G}$ and $\mathrm{S}$ being a $\tau$ - set we have $|S| \leq k$. Hence $|S|=k$. That is $\tau(G)=k$.

Theorem 1.11. Let $G$ is transitively traced and let $V(G)$ is partitioned into $V_{1}, V_{2}, \ldots, V_{k}$ such that the graph $\left\langle V_{i}\right\rangle$ induced by each $V_{i}$ is maximal track connected subgraph of $G$. If $V_{1}, V_{2}, \ldots, V_{k}$ have $m_{1}, m_{2}, \ldots, m_{k}$ vertices respectively, then $G$ has $m_{1} m_{2} \ldots m_{k}$ different $\tau$-set..

Proof. Let $\mathrm{G}$ is transitively traced and let $V(G)$ is partitioned into $V_{1}, V_{2}, \ldots, V_{k}$ such that the graph $\left\langle V_{i}\right\rangle$ induced by each $V_{i}$ is maximal track connected subgraph of G. Let $V_{1}, V_{2}, \ldots, V_{k}$ have $m_{1}, m_{2}, \ldots, m_{k}$ vertices respectively. Then a set $\mathbf{S}$ of vertices which contains exactly one element from each $V_{i}$ is form a $\tau$ set and it can be chosen in $m_{1} m_{2} \ldots m_{k}$ ways.

Theorem 1.12. Let $G(V, E)$ be a transitively tracked graph, $S^{*}$ be a cycle tracking set and $v \in S^{*}$. Then $p t\left[v, S^{*}\right]=\{v\}$ for a cycle tracking set $S^{*}$ if and only if $v$ belongs to every cycle tracking set $S$ and $p t[v, S]=\{v\}$

Proof. Let $G(V, E)$ be a transitively tracked graph and $v \in V$. Let $S^{*}$ be a cycle tracking set containing $v$ with $p t\left[v, S^{*}\right]=\{v\}$. Let $S$ be any cycle tracking set. Then there exists a vertex $u \in S$ such that $v \in T_{G}(u)$. Since $S^{*}$ is a cycle tracing set there exists a vertex $w \in S^{*}$ such that $u \in T_{G}(w)$. Since $\mathrm{G}$ is transitively tracked $v \in T_{G}(w)$. This is possible if and only if $w=u=v$. Hence $v \in S$ and $p t[v, S]=\{v\}$.

If $\mathrm{G}$ is not transitively tracked then the above theorem may not hold. In figure 2, $S=\left\{v_{4}, v_{5}, v_{9}, v_{14}\right\}$ is a cycle tracking set of $\mathrm{G}$ and $p t\left[v_{5}, S\right]=v_{5}$. But $S^{*}=\left\{v_{2}, v_{4}, v_{9}, v_{14}\right\}$ is another cycle tracking sets of $\mathrm{G}$ not containing $v_{5}$.

Theorem 1.13. [3] Let $G(V, E)$ be any graph. Then the following statements are equivalent.

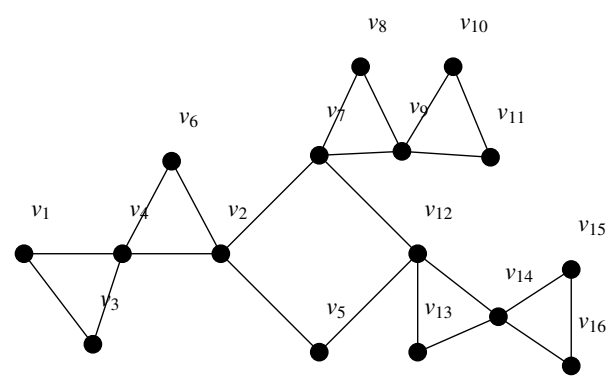

$\mathrm{G}$

Figure 2. G

(i) A vertex $v$ is in every cycle tracking sets.

(ii) $v \in S$ for all cycle tracking set $S$ and $p t[v, S]=\{v\}$.

(iii) $v$ is a trace free vertex.

By Theorem 1.13 and Theorem1.12 we have the Corollary

Corollary 1.14. In a transitively tracked graph $G(V, E)$, $p t[v, S]=\{v\}$ for any cycle tracking set $S$ if and only if $v$ is a trace free vertex.

Theorem 1.15. Let $G(V, E)$ be a graph. If $t=T$ then $G$ is transitively tracked.

Proof. Let $G(V, E)$ be a graph. Suppose $t=T$. Let $v_{1} \in$ $T_{G}\left(v_{2}\right)$. We have $\left|T_{G}\left(v_{1}\right)\right|=\left|T_{G}\left(v_{2}\right)\right|=t$. We claim that $T_{G}\left(v_{1}\right)=T_{G}\left(v_{2}\right)$. If not, $v_{2}$ is a cut vertex. Therefore there exists a vertex $v_{3} \in T_{G}\left(v_{2}\right)$ such that $v_{3} \notin T_{G}\left(v_{1}\right)$. But since $\left|T_{G}\left(v_{2}\right)\right|=\left|T_{G}\left(v_{3}\right)\right|$ and $T_{G}\left(v_{2}\right) \neq T_{G}\left(v_{3}\right)$ there exist a vertex $v_{4} \in T_{G}\left(v_{3}\right)$ such that $v_{4} \notin T_{G}\left(v_{2}\right)$. Then $v_{4} \notin T_{G}\left(v_{1}\right)$ (Otherwise there exist two internally disjoint paths from $v_{1}$ to $v_{4}$. Since there is a path from $v_{1}$ to $v_{4}$ containing both $v_{2}$ and $v_{3}$, and since $v_{2}$ and $v_{3}$ are cut vertices it is not possible.). Continuing in this manner we get a sequence of vertices $v_{1}, v_{2}, \ldots, v_{n}, \ldots$ such that $v_{n} \in T_{G}\left(v_{n-1}\right), v_{n} \notin T_{G}\left(v_{i}\right)$ for $1 \leq i \leq n-2$. But it is not possible as $\mathrm{G}$ is a finite graph. So $T_{G}\left(v_{1}\right)=T_{G}\left(v_{2}\right)$.

Theorem 1.16. Let $G(V, E)$ be a graph. Then $t=T=n$ if and only if $G$ is track connected.

Proof. Let $G(V, E)$ be a graph. Suppose $t=T=n$. Then $T_{G}(v)=V$ for all $v \in V$. That is for every $u, v \in G, u$ and $v$ belong to same cycle and hence there exist two distinct paths from $\mathrm{u}$ to v. So $\mathrm{G}$ is track connected.

Conversely suppose that $\mathrm{G}$ is track connected. Then there exist two distinct paths from any two vertices $\mathrm{u}$ to $\mathrm{v}$. That is $u$ and $v$ belong to a common cycle and hence $T_{G}(v)=V$ for all $v \in V$. Thus $t=T=n$.

Definition 1.17. A graph $G$ is well tracked if $\tau(G)=T(G)$ 
Theorem 1.18. Let $G(V, E)$ be a graph. If $V$ can be partitioned in to maximal track connected subset of $G$, then $G$ is well tracked.

Proof. Let $V_{1}, V_{2}, \ldots, V_{k}$ be a partition of $\mathrm{V}$ into maximal track connected sets. Let $v_{i} \in V_{i}, \mathrm{i}=1,2, \ldots, \mathrm{k}$. Since $V_{i}$ is the only maximal track connected set containing $v_{i}, v_{i}$ can track all elements of $V_{i}$ and no element of $V_{j}$ for $i \neq j$. So $\left\{v_{1}, v_{2}, \ldots, v_{k}\right\}$ forms a minimal cycle tracking set. In fact any such minimal cycle tracking set is obtained like this. Hence $\tau(G)=T(G)$ and $\mathrm{G}$ is well tracked.

Corollary 1.19. If $G$ is transitively tracked then $G$ is well tracked.

The converse of Corollary 1.19 need not be true as $C_{5} \circ K_{2}$ is well tracked but not transitively tracked. A graph $\mathrm{G}$ is said to be trace excellent if every vertex belong to some $\tau$ - set.

Definition 1.20. Two vertices $u, v$ of a graph $G$ are said to be trace independent if $u \notin T_{G}(v)$.

ie; two vertices $u, v$ of a graph $G$ are said to be trace independent if $u$ and $v$ are not vertices of same cycles of $G$.

Definition 1.21. A set $S$ of vertices in a graph $G(V, E)$ is called a trace independent set if any two vertices of $S$ are trace independent in $G$.

Theorem 1.22. [3] For any graph $G, \tau(G) \leq \tau_{i}(G) \leq T_{i}(G) \leq$ $T(G)$.

The Theorem 1.23 follows from Theorem 1.22 and by Corollary 1.19

Theorem 1.23. For a transitively tracked graph $G \tau(G)=$ $\tau_{i}(G)=T_{i}(G)=T(G)$.

Definition 1.24. Let $G(V, E)$ be a graph, a set $X$ subset of $V$ is said to be track closed if for every pair of vertices $u, v \in X$ $u \in T_{G}(v)$.

Definition 1.25. A track closed set is maximal track closed set if no proper super set $S^{\prime} \supset S$ is a track closed set.

1. Every subset of a track closed set is track closed set.

2. Vertex set of a track connected subgraph of a graph is track closed.

Theorem 1.26. Let $G$ be a transitively tracked graph. $X \subset V$ is track closed if and only if the graph induced by $X$ is a subgraph of a maximal track connected subgraph of $G$.

Proof. Let $\mathrm{G}$ be a transitively tracked graph. Suppose $X \subset$ $V$ is track closed. Then for every pair of vertices $u, v \in X$ $u \in T_{G}(v)$. By Theorem 1.4 for every pair of vertices $u, v \in X$ $T_{G}(u)=T_{G}(v)$. Hence $X \subset T_{G}(v)$ and by Theorem 1.6 the graph induced by $T_{G}(v)$ is maximal track connected. Hence the graph induced by $\mathrm{X}$ is a subgraph of a maximal track connected subgraph of $\mathrm{G}$.
Conversely suppose that the graph induced by $X$ is a subgraph of a maximal track connected subgraph $H$ of G. Then $V(H)$ is track closed. Hence its subset $X$ is also track closed.

Corollary 1.27. Let $G$ be a transitively tracked graph. Then the maximal track closed sets of $G$ are precisely the maximal track connected subgraph of $G$.

Corollary 1.28. A graph $G(V, E)$ is a transitively tracked graph if only if $V(G)$ can be partitioned into $V_{1}, V_{2}, \ldots, V_{k}$ each $V_{i}$ is a maximal track closed subset of $V(G)$.

Definition 1.29. We call a vertex that is contained in some $\tau$ - set of a graph $G$ is a trace good vertex, otherwise it is a trace bad vertex. Let $g t(G)$ (respectively, bt $(G))$ denote the number of trace good (respectively,trace bad) vertices in a graph $G$.

Definition 1.30. A graph $G$ is $\tau$-excellent if each of its vertex belongs to some $\tau$-set of $G$.

i.e if $\mathrm{G}$ is $\tau$ - excellent then $\mathrm{g}(\mathrm{G})=\mathrm{n}$.

Theorem 1.31. If a graph $G$ is transitively traced then $G$ is trace excellent.

Proof. Let $\mathrm{G}$ be a transitively tracked graph. Let $\mathrm{S}$ be a $\tau-$ set of G. let $v \in V$. Then there exists a $u \in S$ such that $u \in$ $T_{G}(v)$. Since $\mathrm{G}$ is transitively tracked $T_{G}(u)=T_{G}(v)$. Hence $S \backslash\{u\} \cup\{v\}$ is a $\tau$-set containing $v$. Since $v$ is arbitrary G is trace excellent.

The converse may not be true. $C_{5} \circ K_{2}$ is trace excellent but not transitively tracked.

Definition 1.32. Let $G(V, E)$ be any graph. A cycle tracking set $S \subset V(G)$ is said to be efficient cycle tracking set if $\mid T_{G}(v) \cap$ $S \mid=1$ for every $v \in V(G)$.

Theorem 1.33. Let $G$ be a transitively tracked graph. Then every minimal cycle tracking set is efficient cycle tracking set of $G$.

Proof. Let $\mathrm{G}$ be a transitively tracked graph. Let $\mathrm{S}$ be a minimal cycle tracking set of G. Let $v \in V(G)$. Then there exist a vertex $u \in S$ such that $v \in T_{G}(u)$. Hence $\left|T_{G}(v) \cap S\right| \geq 1$. If possible assume that there are two vertices $w_{1}, w_{2} \in T_{G}(v) \cap$ $S$. Then by Theorem $1.4 T_{G}\left(w_{1}\right)=T_{G}\left(w_{2}\right)=T_{G}(v)$. Then $S \backslash w_{1}$ form a cycle tracking set of G,a contradiction to the minimality of S. So $\left|T_{G}(v) \cap S\right|=1$.

\section{References}

[1] Harary F,'Graph Theory",Adison-Wesley.

[2] Kirchhoff, G. (1847) "Über die Auflösung der Gleichungen, auf welche man bei der Untersuchung der linearen Verteilung galvanischer Ströme geführt wird" (On the solution of the equations to which one is led during the investigation of the linear distribution of galvanic currents), Annalen der Physik und Chemie, 72 (12) : 497-508 
[3] Jalsiya M.P. and Raji Pilakat, "Cycle tracking sets of a graph: An efficient approach to circuit analysis", to appear.

[4] Jalsiya M.P. and Raji Pilakat, "Independent and Irredundant Cycle Tracking Sets of a Graph : An efficient approach to electrical circuit analysis", to appear.

[5] James Clerk Maxwell, A Treatise on Electricity and Magnetism (Oxford, England: Clarendon Press, 1873), vol. 1, Part II, "On linear systems of conductors in general", pp. 333-336.

[6] Wataru Mayeda and Sundaram Seshu (November 1957) "Topological Formulas for Network Functions," University of Illinois Engineering Experiment Station Bulletin, no. 446, p. 5.

[7] H. Poincaré (1900) "Second complément à l'Analysis Situs", Proceedings of the London Mathematical Society, 32 : 277-308. Available on-line at: Mocavo.com

[8] D.B.West,'Introduction to Graph Theory", 2nd ed. Pearson Education.

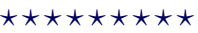

ISSN(P):2319-3786

Malaya Journal of Matematik

$\operatorname{ISSN}(\mathrm{O}): 2321-5666$

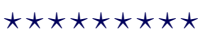

